\title{
KLHL22 promotes malignant melanoma growth in vitro and in vivo by activating the PI3K/Akt/mTOR signaling pathway
}

\author{
X.R. LIU ${ }^{1, *}$, W. WANG ${ }^{2, *}$, H.M. LI ${ }^{3, *}$ \\ ${ }^{1}$ Department of Dermatology, Clinical Center of Spaceport, Chinese PLA General Hospital, Beijing 10094, China; ${ }^{2}$ Department of Dermatology, \\ No. 929 Hospital of the Chinese PLA, Shanghai 200433, China; ${ }^{3}$ Department of General Practice, Clinical Center of Spaceport, Chinese PLA \\ General Hospital, Beijing 10094, China
}

*Correspondence: Lihm2019@126.com

Received September 23, 2019 / Accepted January 31, 2020

\begin{abstract}
The kelch like family member 22 (KLHL22) is a member of the KLHL (Kelch-like) gene family, which was involved in the progression of breast cancer. However, its role remains unclear in malignant melanoma (MM). Our study found that KLHL22 expression was upregulated in human MM tissues. Regarding the functional analysis for KLHL22 in the progression of MM cells, we demonstrated that overexpression of KLHL22 could promote MM cell growth in vitro. Vice versa, knockdown of KLHL22 could suppress the proliferation of MM cells. Furthermore, KLHL22 also promoted tumorigenesis of $\mathrm{MM}$ cells in vivo. In experiments investigating the underlying mechanism, expressions of p-Akt and p-mTOR were significantly increased by overexpression of KLHL22. Meanwhile, knockdown of KLHL22 could decrease the expression levels of p-Akt and p-mTOR. Our studies thus suggest that KLHL22 can promote the growth of MM cells via activating the $\mathrm{PI} 3 \mathrm{~K} / \mathrm{Akt} / \mathrm{mTOR}$ signaling pathway, which can serve as a potential target in the diagnosis and/or treatment of MM.
\end{abstract}

Key words: KLHL22, growth, melanoma, PI3K/Akt/mTOR

Malignant melanoma (MM) is an aggressive skin cancer that accounts for $80 \%$ of deaths caused by skin cancer [1, 2]. The incidence of MM has risen steeply by approximately 3\% annually around the world [3]. Despite the significant advances obtained in diagnosis and treatments of MM, the prognosis of MM remains particularly suboptimal due to its propensity to metastasize and it still causes nearly 50,000 deaths per year worldwide [4]. MM is difficult to treat through numerous treatments such as surgery, radiation therapy, or chemotherapy. Therefore, a better understanding of the underlying molecular mechanism of MM is important to develop new therapeutic targets for MM patients.

The kelch like family member 22 (KLHL22), as a member of the KLHL (Kelch-like) gene family, is located at 22q11.21. The KLHL gene family encodes proteins that constitute a subgroup at the intersection between the $\mathrm{BTB} / \mathrm{POZ}$ domain and Kelch domain superfamilies. KLHL22 is a BTB (Bricá-brac-Tramtrack-Broad) adaptor protein, usually forming a functional cullin-RING E3 ubiquitin ligase complex with the scaffold protein CUL3 and the ring-finger protein RBX1 $[5,6]$. Some KLHL family members such as KLHL6, KLHL19, KLHL20, and KLHL37 are reported to be associated with cancer [7-10]. Besides, Chen et al. demonstrated that depletion of KLHL22 in breast cancer cells suppresses tumor growth in nude mice and pharmacological interventions targeting KLHL22 may have therapeutic potential for the treatment of breast cancer and age-related diseases [11]. However, the role of KLHL22 in MM still remained unclear.

In our study, we focused on the importance of KLHL22 in $\mathrm{MM}$ growth in vitro and in vivo. We demonstrated that KLHL22 expression was upregulated in human MM tissues. KLHL22 promoted the growth of MM cells in vitro and in vivo. Additionally, we explored the underlying molecular mechanism of KLHL22 in MM progression.

\section{Patients and methods}

Melanoma tissue samples. After obtaining written informed consent, thirty-five pairs of primary MM tissues and their paired adjacent normal tissues were obtained from patients who underwent surgery and without pre-operative radiation or chemotherapy at the Chinese PLA General Hospital, Beijing, China. All tissue specimens were immediately frozen in liquid nitrogen after surgery. The samples were subjected to RNA extraction for quantitative real time PCR (qPCR) analysis. Our study was approved by the Ethics 
Committee of the Institute for the Chinese PLA General Hospital, Beijing, China.

RNA preparation and $\mathbf{q P C R}$. Total RNA of 35 pairs of tumor tissues and adjacent normal tissues, cell lines, and xenograft tumors samples was extracted using the Trizol reagent (Invitrogen). $2 \mu \mathrm{g}$ of total RNA was reversetranscribed to cDNA with the PrimeScript RT reagent Kit (Takara) according to the manufacturer's instructions. SYBR Green qPCR SuperMix (Invitrogen) was used for qPCR. The thermocycling protocol of qPCR was: preheating at $95^{\circ} \mathrm{C}$ for $10 \mathrm{~min}$, followed by 40 cycles of denaturation at $95^{\circ} \mathrm{C}$ for $20 \mathrm{~s}$ and extension at $60^{\circ} \mathrm{C}$ for $30 \mathrm{~s}$. The KLHL22-specific primers were 5'-ATCCACGGTGTGTCCTACAAT-3' (forward) and 5'-GCCGGTAGACATCGAGAATGTT-3' (reverse), and the GAPDH primers were 5'ATGACCCCTTCATTGACCTCA-3' (forward) and 5'-GAGATGATCACCCTTTTGGCT-3' (reverse). The relative expression of KLHL22 was normalized to GAPDH.

Cell culture. Human MM cell lines A375, HS944T, HS294T, and SKMEL5 were purchased from the cell bank of the Shanghai Biology Institute, Chinese Academy of Science (Shanghai, China). All cell lines were cultured in DMEM medium with supplemented $10 \%$ fetal bovine serum (Gibco), 100 units/ml penicillin, and $100 \mathrm{mg} / \mathrm{ml}$ streptomycin. Cell lines were maintained at $37^{\circ} \mathrm{C}$ in a humidified incubator containing $5 \% \mathrm{CO}_{2}$.

Plasmid construction and cell transfection. The coding sequence for the full length of KLHL22 was cloned into the p23-ZsGreen plasmid (Invitrogen) to generate the KLHL22 expression vector. Lentiviral short hairpin RNA (shRNA) targeting KLHL22 were designed using software provided by Qiagen (Valencia, CA, USA) and inserted into pLKO.1-TRC vector. A375 and HS944T cells were infected with p23-ZsGreen-KLHL22 lentiviral; HS294T and SKMEL5 cells were infected with pLKO.1shKLHL22. Overexpressed and silenced cells were sorted using flow cytometry or selected by puromycin $(4 \mu \mathrm{g} / \mathrm{ml})$ for 4 days, respectively. The sequences of the shKLHL22-1 were 5'-CCGGCTACAATGCTATGTGCCAAATCTCGAGATTTGGCACATAGCATTGTAGTTTT TG-3' (forward) and 5'-AATTCAAAAACTACAATGCTATGTGCCAAATCTCGAGAT T TGGCACATAGCAT T GTAG-3' (reverse). The sequences of the shKLHL22-2 were: 5'-CCGGCCAGAGTTTAGAAGTATTGAACTCGAGT TCAATACT TCTAAACTCTGGTTT T TG-3' (forward) and 5'-AATTCAAAAACCAGAGTTTAGAAGTATTGAACTCGAGTTCAATACTTCTAAACTCTGG-3' (reverse). Overexpressed KLHL22 with a Flag tag was detected in cell lines with an anti-Flag antibody (Sigma).

Western blot. Total cells were lysed in RIPA Lysis Buffer and PMSF (Thermo Scientific, USA), and the lysates were centrifuged at $10000 \times \mathrm{g}$ for 15 minutes at $4{ }^{\circ} \mathrm{C}$ according to the manufacturer's instructions. Protein concentration was determined using the Bradford reagent (Sigma). The lysates were separated on $10 \%$ SDS-PAGE, and blots were immunob- lotted with indicated primary antibodies and the corresponding horseradish peroxidase-conjugated secondary antibodies. Primary antibodies against the following proteins were used in this study: KLHL22 (1:1000, 16214-1-AP), phosphorylated and total forms of Akt (1:1000, 10176-2-AP, 66444-1-Ig) and GAPDH (1:1000, 10494-1-AP) purchased from ProteinTech; phosphorylated and total forms of mTOR (1:1000, sc-517464, sc-293133) were purchased from Santa Cruz Biotechnology; Flag (1:4000, F7425) was purchased from Sigma.

Cell proliferation analysis. Cell proliferation was detected with crystal violet assay or MTT staining method described by the manufacturer. 1000 cells were seeded into 6-well plates and the cells were cultured in medium with $10 \%$ FBS with the medium change every three days. 10 days later, the medium was removed, and the cells were stained with $1 \mathrm{ml} 0.5 \%$ crystal violet solution in $20 \%$ methanol. After staining for $10 \mathrm{~min}$, the fixed cells were washed with phosphate-buffered saline (PBS) and photographed. Then, 1 $\mathrm{ml}$ glacial acetic acid was added to the cells and the optical density (OD) was detected at $600 \mathrm{~nm}$ by a microplate reader.

In the MTT assay, 1000 cells were seeded into 96-well plates, and cell viability was detected by MTT, after 1, 2, 3, 4 , 5, or 6-day incubation, $20 \mu \mathrm{l}$ of a $5 \mathrm{mg} / \mathrm{ml}$ MTT solution was added to each well, the plate was further incubated at $37^{\circ} \mathrm{C}$ for $4 \mathrm{~h}$. Thereafter the medium was aspirated and the wells washed with PBS, drained for approximately $2 \mathrm{~h}$, and then the solution was carefully aspirated and $200 \mu \mathrm{DMSO}$ was added to dissolve the crystal. The microtiter plate was placed on a shaker to dissolve the dye. The absorbance was measured at $490 \mathrm{~nm}$ using a microplate reader.

Tumorigenesis in vivo. All male nude mice were raised at the specific pathogen-free animal center. A375 control and overexpression cell suspensions $\left(1 \times 10^{6}\right.$ cells/100 $\left.\mu \mathrm{l}\right)$ were injected subcutaneously on the flanks of the 5 -week old male nude mice at two sites ( 4 mice in each group). Tumor volume $\left(\mathrm{mm}^{3}\right)$ was measured each week. The tumor volume was calculated as $\mathrm{mm}^{3}=0.5 \times$ length $\times$ width $^{2}$. At the end of the experiment, and the tumors were excised, photographed, and weighted. All animal experiments were performed under the approval of the Institutional Animal Care and Use Committee (IACUC) of the Chinese PLA General Hospital, Beijing, China.

Statistical analysis. All data were processed with GraphPad Prism 5 (GraphPad Software Inc., CA, USA), and results were expressed as mean \pm standard deviation. The comparison between the two groups was conducted using the Student's test. A p-value $<0.05$ indicated a significant statistical difference and is marked with an asterisk.

\section{Results}

KLHL22 is upregulated in MM clinical samples. To explore the potential roles of KLHL22 in MM, we examined its expression in 35 pairs of clinical MM tissues and their 
A

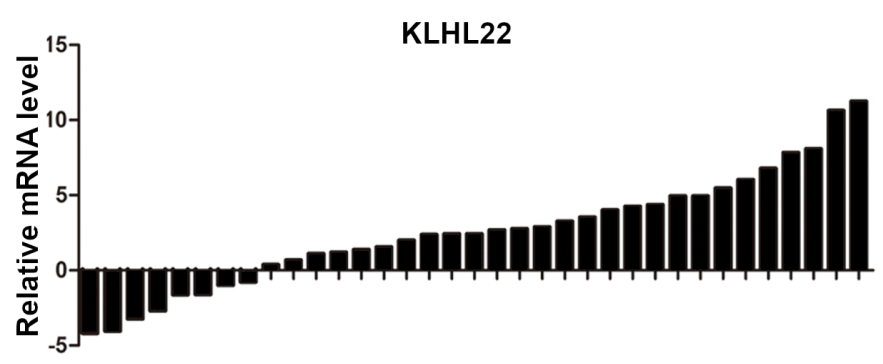

B



C

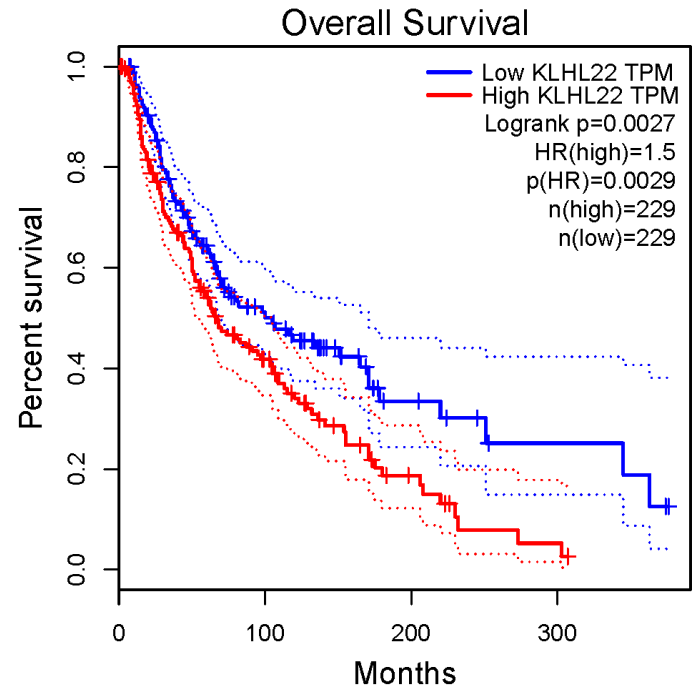

Disease Free Survival

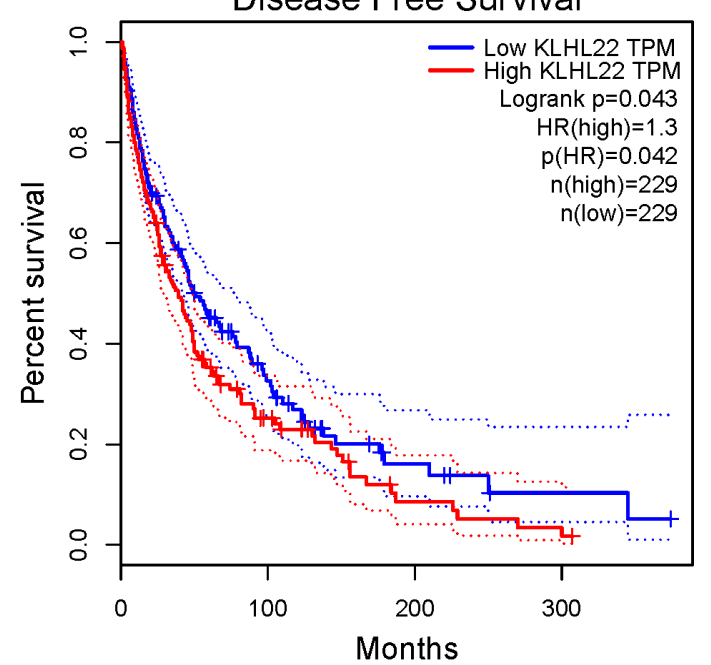

Figure 1. KLHL22 expression was increased in MM tissues and correlated with MM patient survival. A) KLHL22 mRNA levels in 35 pairs of tumor samples $(\mathrm{T})$ and matched normal tissues $(\mathrm{N})$ determined by q-PCR. B) The expression of KLHL22 was higher in tumor (T) tissues than normal (N) tissues derived from the GEPIA database. SKCM, Skin Cutaneous Melanoma. C) High KLHL22 expression correlated with worse overall survival in MM patients derived from the GEPIA database. D) High KLHL22 expression correlated with worse disease-free survival in MM patients derived from the GEPIA database.

corresponding normal tissue at the mRNA level, which showed that the mRNA expression of KLHL22 was significantly upregulated in 27 of 35 (77\%) MM tissues compared to the paired normal tissues (Figure 1A). Based on the GEPIA database, we also found that the expression of KLHL22 was significantly higher in the 461 tumor tissues than the 558 normal tissues (Figure 1B). In addition, the overall survival (OS) rate and disease-free survival (DFS) rate were significantly better in the KLHL22-low group compared to the KLHL22-high group $(\mathrm{p}=0.0027$ and 0.043 , respectively; Figures 1C, 1D). Taken together, we can confirm that KLHL22 is upregulated in MM tissues based on our analysis and GEPIA database.
Successful overexpression and knockdown of KLHL22 in MM cells. Based on the previous clinical analysis, we speculated that KLHL22 might influence the growth of MM cells. Firstly, we detected the KLHL22 expression levels in several human MM cell lines including A375, HS944T, HS294T, and SKMEL5 by western blot and qPCR. We found that KLHL22 was highly expressed in HS294T and SKMEL5 cells than A375 and HS944T cells both as mRNA and protein levels (Figures 2A, 2B). To further explore the function of KLHL22 in MM cells, we transfected the A375 and HS944T cells which have relatively low expression of KLHL22 with plasmids containing either an empty p23 vector or KLHL22 overexpression vector (Figures 2C, 2D). And, we knocked 
down the expression of KLHL22 in HS294T and SKMEL5 MM cells which have relatively high expression of KLHL22 (Figures 2E, F).

KLHL22 overexpression promoted the growth of MM cells in vitro. The MTT assays showed that the absorbance values of the A375 and HS944T MM cells at 4, 5, and 6-day after transfection with KLHL22 overexpression vectors were significantly higher than those of the untreated cells (Figures 3A, 3D). In addition, the crystal violet assays showed that KLHL22 overexpression significantly promoted proliferation in A375 and HS944T cells (Figures 3B, 3E). And the absorbance values of the A375 and HS944T cells after transfection with KLHL22 overexpression vectors were significantly higher than those of the untreated cells (Figures 3C, $3 \mathrm{~F})$.

The knockdown of KLHL22 inhibited the growth of MM cells in vitro. In HS294T and SKMEL5 cells, we knocked down the basal expression of KLHL22 by shRNA. Similar to the overexpression experiments, we explored the growth of the control and shRNA-KLHL22 cell lines. The MTT assays showed that the absorbance values of the HS294T and SKMEL5 cells at day 6 after KLHL22 knockdown were significantly lower than those of the untreated cells (Figures 4A, 4D). The crystal violet assays found that knockdown of KLHL22 significantly inhibited the proliferation of HS294T and SKMEL5 cells (Figures 4B, 4E). Furthermore, the absorbance values of crystal violet in the HS294T and SKMEL5 cells after KLHL22 knockdown were significantly lower than those of the untreated cells (Figures 4C, 4F).

KLHL22 promoted tumorigenesis of MM cells in vivo. Based on the results of in vitro experiments, control and KLHL22 overexpression A375 cells were injected subcutaneously into the flanks of nude mice and the tumors were harvested and photographed. Consistent with in vitro study, KLHL22 overexpression in A375 cells dramatically promoted tumor growth compared with the control cells in the xenograft mouse model (Figure 5A). Moreover, the relative mRNA expression of KLHL22 was higher in KLHL22 overexpression compared to the Vector group (Figure 5B). The tumors were heavier and grew more quickly in the
A

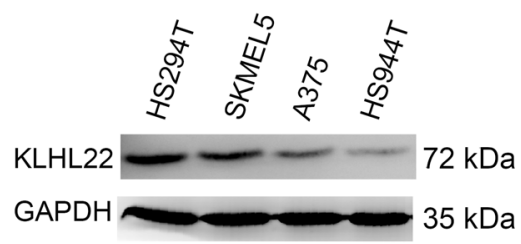

C

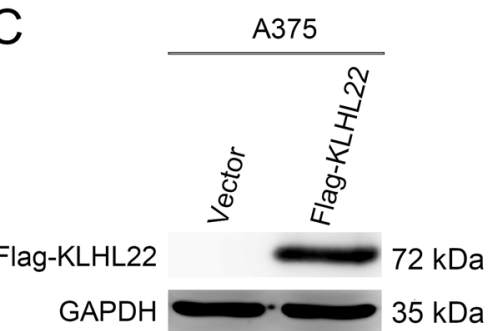

$E$

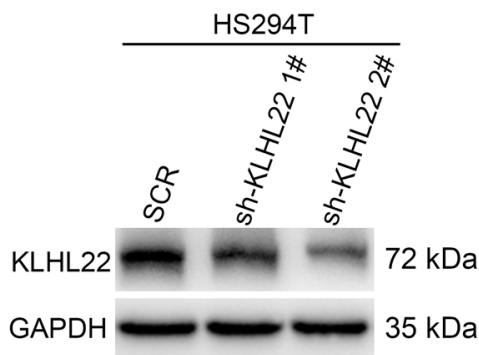

B

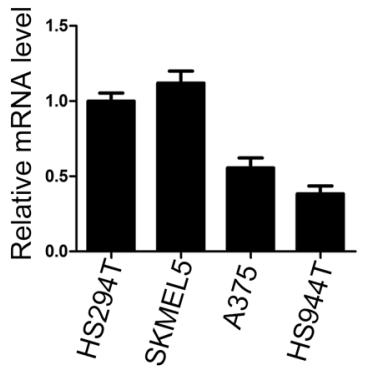

D

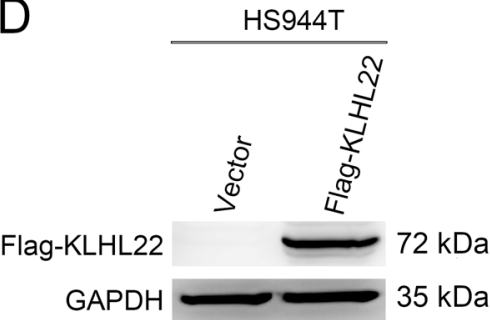

$\mathrm{F}$



Figure 2. Overexpression and knockdown of KLHL22 in MM cell lines. A) Western blotting showing KLHL22 expression in four MM cell lines. GAPDH was used as a loading control. B) KLHL22 mRNA levels in four MM cell lines determined by q-PCR. Western blotting showing the overexpression of KLHL22 in A375 (C) and HS944T (D) cells. Western blotting showing the knockdown of KLHL22 in HS294T (E) and SKMEL5 (F) cells. 
A

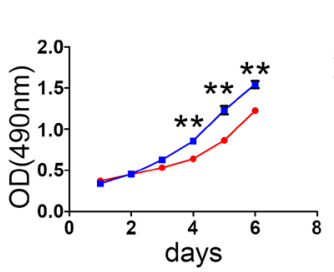

B

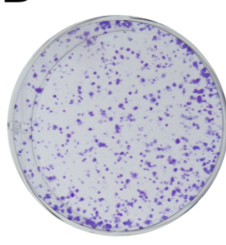

Vector

A375

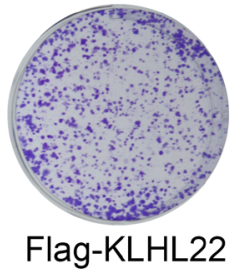

C

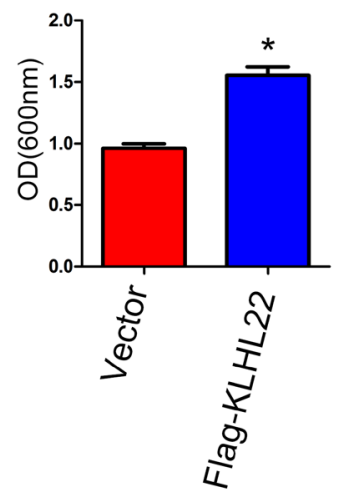

D HS944T

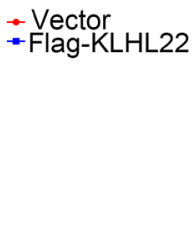

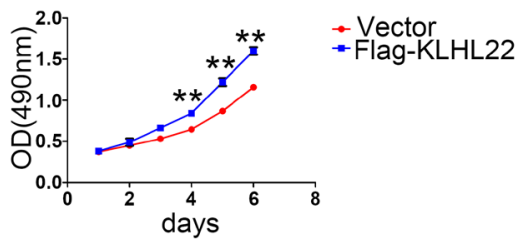

E

HS944T

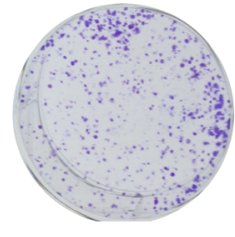

Vector

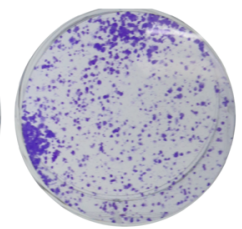

Flag-KLHL22

F

HS944T

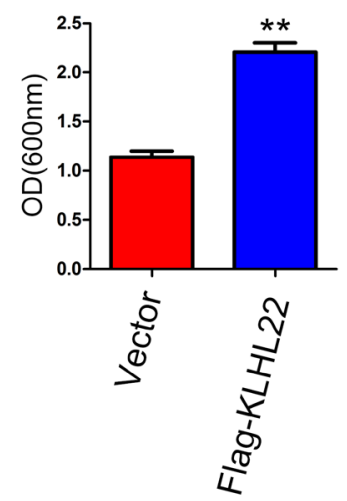

Figure 3. KLHL22 overexpression promoted growth in MM cells. The effects of KLHL22 overexpression on the viability of A375 (A) and HS944T (D) cells were assessed by MTT assays. The effects of KLHL22 overexpression on the viability of A375 (B) and HS944T (E) cells were assessed by crystal violet assays. The OD value of crystal violet assays in A375 (B) and HS944T (E) cells. Data are presented as mean \pm SD of three independent experiments. ${ }^{\star} \mathrm{p}<0.05,{ }^{\star *} \mathrm{p}<0.01,{ }^{\star * *} \mathrm{p}<0.001$

KLHL22 overexpression group than those generated by the control cells $(n=4 ; p<0.001$; Figures $5 C$, 5D). These results indicated that KLHL22 may act as an oncogene that promotes tumorigenesis of MM cells in vivo.

KLHL22 activated the PI3K/Akt/mTOR signaling pathway in MM cells. It has been reported that PI3K/Akt/ mTOR signaling pathway plays an important role in the development and progression of MM [12-14]. Thus, the western blotting analysis was carried out to investigate the expression levels of Akt, p-Akt, mTOR, and p-mTOR in KLHL22 overexpression and knockdown cell lines. In the KLHL22 overexpression group of A375 and HS944T cells, the total expressions of Akt and mTOR showed no obvious difference compared with the control group, whereas there was a marked increase in expressions of p-Akt and p-mTOR compared with the control group (Figure 6A). On the contrary, the knockdown of KLHL22 exhibited a significant decrease in expressions of p-Akt and p-mTOR compared with the control group in HS294T and SKMEL5 (Figure 6B). Besides, based on the correlation analysis of the GEPIA database, the expression level of KLHL22 was positively correlated with that of $\mathrm{p}$-Akt and p-mTOR (Figures 6C, 6D). In summary, these results indicated that KLHL22 could activate the PI3K/Akt/mTOR signaling pathway.

\section{Discussion}

The prognosis of MM, over the past few decades, has been relatively poor, without a highly sensitive and specific, early diagnostic method. Surgical and radiation therapies are the 
A

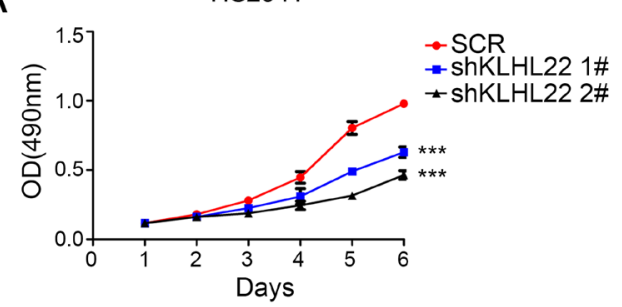

B



C

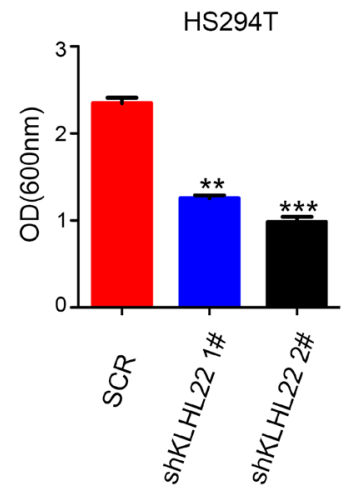

$\mathrm{D}$



E



$\mathrm{F}$

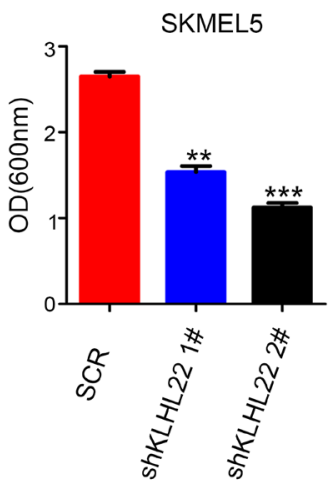

Figure 4. KLHL22 knockdown inhibited growth in MM cells. The effects of KLHL22 knockdown on the viability of HS294T (A) and SKMEL5 (D) cells were assessed by MTT assays. The effects of KLHL22 knockdown on the viability of HS294T (B) and SKMEL5 (E) cells were assessed by crystal violet assays. The OD value of crystal violet assays in HS294T (C) and SKMEL5 (F) cells. Data are presented as mean \pm SD of three independent experiments. ${ }^{\star} \mathrm{p}<0.05,{ }^{* *} \mathrm{p}<0.01,{ }^{* * *} \mathrm{p}<0.001$

A

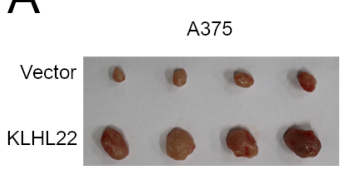

C

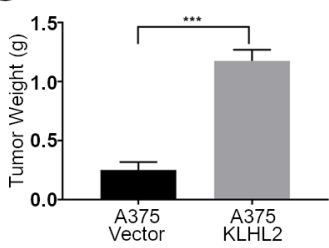

B

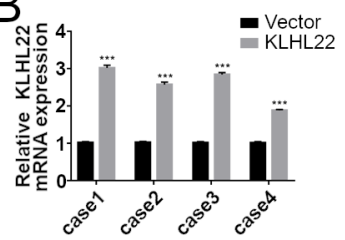

D

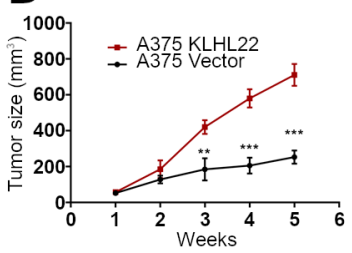

Figure 5. KLHL22 promoted tumorigenesis of MM cells in vivo. A) Representative images of the tumors generated by KLHL22 con and overexpression A375 cells. B) KLHL22 mRNA expression in Vector and KLHL22 overexpression tumor samples determined by q-PCR. C) Tumor weight of the tumors (g). D) Growth curves of the tumors $\left(\mathrm{mm}^{3}\right)$. Data are presented as mean $\pm \mathrm{SD}$ of three independent experiments. ${ }^{\star} \mathrm{p}<0.05$, ${ }^{* *} \mathrm{p}<0.01,{ }^{* * *} \mathrm{p}<0.001$

main forms of treatment for MM; however, many patients eventually develop into the advanced stages of the disease. Our study aimed to find new potential intervention targets for MM. In recent years, emerging evidence has confirmed KLHL22 to be involved in tumorigenesis and cancer progression. As reported, KLHL22 can suppress tumor growth of breast cancer cells.

In the current study, we firstly investigated the KLHL22 expression pattern based on clinical samples and the GEPIA database. Our study found that the KLHL22 was upregulated in human MM tissues compared with the paired normal tissues. Based on the GEPIA database, MM patients with high KLHL22 expression had worse overall survival and diseasefree survival rates than those with low KLHL22 expression. In regard to the functional analysis for KLHL22 in the progression of MM cells, we successfully constructed the KLHL22 overexpression and knockdown MM cell lines. We found that overexpression of KLHL22 could promote MM cell growth in vitro. A knockdown of KLHL22 could suppress 

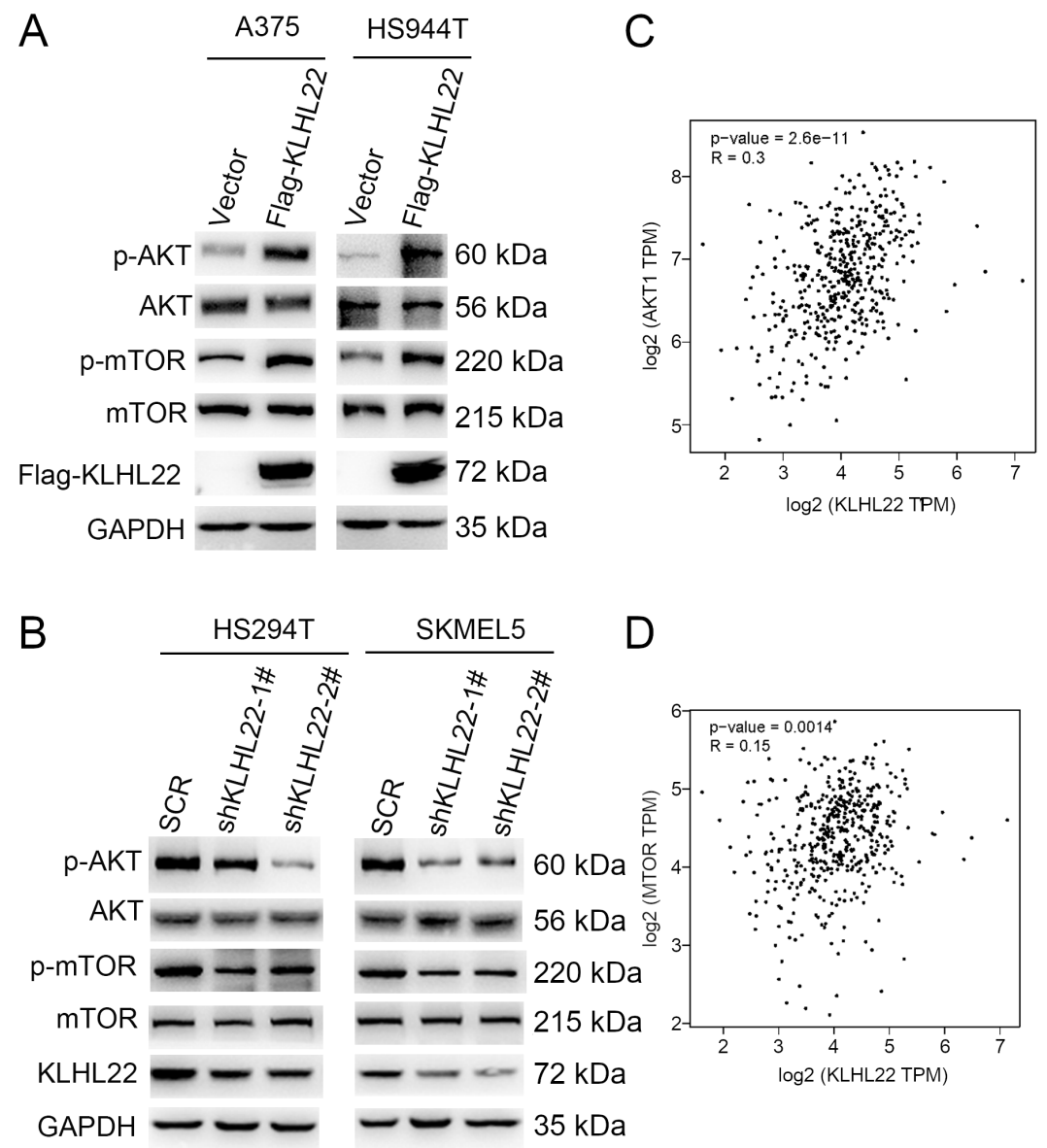

Figure 6. KLHL22 activated the PI3K/Akt/mTOR signaling pathway. The overexpression of KLHL22 respectively activated the expressions of p-Akt and p-mTOR in A375 and HS944T cells according to western blotting (A). GAPDH was used as a loading control. The knockdown of KLHL22 respectively inhibited the expressions of p-Akt and p-mTOR in HS294T and SKMEL5 cells according to western blotting (B). GAPDH was used as a loading control. The expression level of KLHL22 was positively correlated with that of p-Akt (C) and p-mTOR (D) based on the correlation analysis of the GEPIA database.

the proliferation of MM cells. Furthermore, KLHL22 also promoted tumorigenesis of MM cells in nude mice.

In experiments investigating the underlying mechanism, expressions of $\mathrm{p}$-Akt and $\mathrm{p}$-mTOR were significantly increased by overexpression of KLHL22. Meanwhile, knockdown of KLHL22 could decrease the expression levels of p-Akt and p-mTOR. A variety of studies on genetic analyses of genes of multiple cellular signaling pathways reveal that the $\mathrm{PI} 3 \mathrm{~K} / \mathrm{Akt} / \mathrm{mTOR}$ pathway is often highly frequently altered in human cancers [15-17]. In addition, Chen et al. demonstrated that KLHL22 functions as a potential oncogene to active mTORC1 signaling and promote tumor growth in breast cancer [11]. The present study revealed that KLHL22 can promote the growth of MM cells by activating the PI3K/ $\mathrm{Akt} / \mathrm{mTOR}$ signaling pathway. To the best of our knowledge, this is the first study to report how KLHL22 is involved in the growth of MM cells.

In conclusion, our study demonstrated a novel role for KLHL22 in the growth of MM cells via activating the PI3K/
Akt/mTOR signaling pathway. The present results provide a better understanding of the biological function and underlying mechanisms for the role of KLHL22 in tumor development and might serve as a novel therapeutic target for MM.

\section{References}

[1] MILLER AJ, MiHM MC JR. Melanoma. N Engl J Med 2006; 355: 51-65. https://doi.org/10.1056/NEJMra052166.

[2] MALISSEN N, GROB JJ. Metastatic Melanoma: Recent Therapeutic Progress and Future Perspectives. Drugs 2018; 78: 1197-1209. https://doi.org/10.1007/s40265-018-0945-Z

[3] OLSZANSKI AJ. Current and future roles of targeted therapy and immunotherapy in advanced melanoma. J Manag Care Spec Pharm 2014; 20: 346-356. https://doi.org/10.18553/ jmcp.2014.20.4.346

[4] MILLER KD, SIEGEL RL, LIN CC, MARIOTTO AB, KRAMER JL et al. Cancer treatment and survivorship statistics, 2016. CA Cancer J Clin 2016; 66: 271-289. https://doi. org/10.3322/caac. 21349 
[5] PETROSKI MD, DESHAIES RJ. Function and regulation of cullin-RING ubiquitin ligases. Nat Rev Mol Cell Biol 2005; 6: 9-20. https://doi.org/10.1038/nrm1547

[6] DHANOA BS, COGLIATI T, SATISH AG, BRUFORD EA, FRIEDMAN JS. Update on the Kelch-like (KLHL) gene family. Hum Genomics 2013; 7: 13. https://doi.org/10.1186/14797364-7-13

[7] KROLL J, SHI X, CAPRIOLI A, LIU HH, WASKOW C et al. The BTB-kelch protein KLHL6 is involved in B-lymphocyte antigen receptor signaling and germinal center formation. Mol Cell Biol 2005; 25: 8531-8540. https://doi.org/10.1128/ MCB.25.19.8531-8540.2005

[8] SHIBATA T, KOKUBU A, GOTOH M, OJIMA H, OHTA $\mathrm{T}$ et al. Genetic alteration of Keap1 confers constitutive Nrf2 activation and resistance to chemotherapy in gallbladder cancer. Gastroenterology 2008; 135: 1358-1368. https://doi. org/10.1053/j.gastro.2008.06.082

[9] YUAN WC, LEE YR, HUANG SF, LIN YM, CHEN TY et al. A Cullin3-KLHL20 Ubiquitin ligase-dependent pathway targets PML to potentiate HIF-1 signaling and prostate cancer progression. Cancer Cell 2011; 20: 214-228.https://doi. org/10.1016/j.ccr.2011.07.008

[10] LIANG XQ, AVRAHAM HK, JIANG S, AVRAHAM S. Genetic alterations of the NRP/B gene are associated with human brain tumors. Oncogene 2004; 23: 5890-5900. https:// doi.org/10.1038/sj.onc.1207776

[11] CHEN J, OU Y, YANG Y, LI W, XU Y et al. KLHL22 activates amino-acid-dependent mTORC1 signalling to promote tumorigenesis and ageing. Nature 2018; 557: 585-589. https:// doi.org/10.1038/s41586-018-0128-9
[12] LI CY, WANG Q, WANG X, LI G, SHEN S et al. Scutellarin inhibits the invasive potential of malignant melanoma cells through the suppression epithelial-mesenchymal transition and angiogenesis via the PI3K/Akt/mTOR signaling pathway. Eur J Pharmacol 2019; 858: 172463. https://doi. org/10.1016/j.ejphar.2019.172463

[13] LI CY, WANG Q, SHEN S, WEI XL, LI GX. Oridonin inhibits migration, invasion, adhesion and TGF-betal-induced epithelial-mesenchymal transition of melanoma cells by inhibiting the activity of PI3K/Akt/GSK-3beta signaling pathway. Oncol Lett 2018; 15: 1362-1372. https://doi. org/10.3892/ol.2017.7421

[14] LIAO Z, ZHAO J, YANG Y. Downregulation of lncRNA H19 inhibits the migration and invasion of melanoma cells by inactivating the NFkappaB and PI3K/Akt signaling pathways. Mol Med Rep 2018; 17: 7313-7318. https://doi.org/10.3892/ mmr.2018.8782

[15] ALZAHRANI AS. PI3K/Akt/mTOR inhibitors in cancer: At the bench and bedside. Semin Cancer Biol 2019; 59: 125132. https://doi.org/10.1016/j.semcancer.2019.07.009

[16] POLIVKA J JR., JANKU F. Molecular targets for cancer therapy in the PI3K/AKT/mTOR pathway. Pharmacol Ther 2014; 142: 164-175. https://doi.org/10.1016/j.pharmthera.2013.12.004

[17] ARAFEH R, SAMUELS Y. PIK3CA in cancer: The past 30 years. Semin Cancer Biol 2019; 59: 36-49. https://doi. org/10.1016/j.semcancer.2019.02.002 